\title{
Doppler Spectra From a Two-Dimensional Ocean Surface at L-Band
}

\author{
Gabriel Soriano, Maminirina Joelson, and Marc Saillard, Member, IEEE
}

\begin{abstract}
An approximate time-harmonic three-dimensional electromagnetic boundary-integral method, the small-slope integral equation, is combined with a series expansion of the Creamer surface representation at second order with respect to the height, denoted by Creamer (2). The resulting model provides at low numerical cost simulations of the nonlinear ocean surface Doppler spectrum at L-band. As a result of approximations, the model is designed for a low-wind speed, typically up to $5 \mathrm{~m} / \mathrm{s}$. It is shown that applying directly a second-order model such as Creamer (2) to a semiempirical sea surface spectrum induces an unrealistic magnification of small-scale roughness that is involved in the scattering process at microwave frequencies. This paper thus proposes an undressed version of the Pierson-Moskowitz spectrum that corrects this artifact. Full-polarized Doppler simulations at L-band and $70^{\circ}$ incidence are presented. Effects of the surface nonlinearities are outlined, and the simulated Doppler spectra show correct variations with respect to wind speed and direction.
\end{abstract}

Index Terms-Doppler radar, nonlinear wave propagation, remote sensing, sea surface electromagnetic scattering.

\section{INTRODUCTION}

$\mathbf{E}$ LECTROMAGNETIC wave scattering from the sea surface has been intensively studied, experimentally as well as theoretically, for now more than half a century. To take benefit from the fluid motion and get much more information than the average scattering coefficient, one can perform a coherent integration in time with a Doppler radar. Indeed, surface waves moving at different speeds provide different Doppler frequency shifts. This is particularly useful in a monostatic configuration. The square modulus of the time Fourier transform of the complex backcattered field is called the Doppler spectrum and represents the basic information we refer to in this paper.

For the open sea, techniques using coherent microwave radars to retrieve oceanographic information are now well established. In the high-frequency (HF) domain, Crombie [1] explained the Doppler spectrum by the Bragg scattering process. For shorter radar wavelengths, Wright [2] and Bass et al. [3]

Manuscript received August 11, 2005; revised January 16, 2006. This work was supported by the Department Sciences et Technologies de l'Information et de la Communication of Centre National de la Recherche Scientifique in the frame of Equipe Projet Multilaboratoires entitled L-band Active Ocean Remote Sensing.

G. Soriano is with the Université Paul Cezanne, Institut Fresnel Unité Mixte de Recherche (UMR) Centre National de la Recherche Scientifique (CNRS) 6133, 13397 Marseille Cedex 20, France.

M. Joelson is with the Université d'Avignon, Dpt Physique UMR Climat, Solet Environnement (CSE), Faculté des Sciences, Avignon 84000, France.

M. Saillard is with the Université du Sud Toulon-Var, Laboratoire de Sondage Electromagnétique de l'Environnement Terrestre UMR CNRS 6017, La Garde Cedex 83957, France.

Digital Object Identifier 10.1109/TGRS.2006.873580 proposed composite surface models that include hydromodulation of the wavelets by long waves. Concurrently, Barrick and Weber [4], [5] developed a perturbative model, more accurate and analytic, based on a representation of a sea surface up to second order in terms of hydrodynamic combined with a second-order electromagnetic scattering model (SPM2). This SPM2 model is the first one to support the double contribution from nonlinear interactions between water waves and electromagnetic effects. It has been extensively used with a success under various situations for the HF and very high frequency (VHF) bands.

Here, the focus is on the probing of the costal area. To capture small-scale changes that characterize such an environment, one has to raise the frequency up to at least microwaves. As only the gravity waves of the ocean surface are considered, we use the lower part of the microwave band, namely L-band (between 1 and $2 \mathrm{GHz}$, following the IEEE standard radar band nomenclature). From an electromagnetic point of view, standard low-frequency approximations or composite surface models no longer hold at those frequencies, and one has to turn toward more rigorous modelization. Lentz [6] started numerical simulation of the ocean surface by solving the rigorous harmonic boundary value problem. Rino et al. [7] performed Doppler spectrum simulations with surfaces generated according to the nonlinear model by Creamer et al. [8]. More recently, Toporkov and Brown [9], [10] combined the method of ordered multiple interactions with the fast multipole method to address L-band and the low grazing angles. For other nonlinear ocean surface models, see Johnson et al. [11] and Hayslip et al. [12]. However, those numerical simulations cannot predict quantitatively the radar return, since they address a simplified two-dimensional (2-D) representation of the problem. The surface profile is assumed to be invariant along one direction [one-dimensional (1-D) surface], the wind and the radar beam directions being enforced to coincide.

In the present study, we present numerical Doppler spectrum simulations of a 2-D wind-driven sea surface at L-band. To our knowledge, as far as Doppler spectrum simulation is concerned, the paper constitutes the first attempt of a threedimensional (3-D) electromagnetic simulation. Following the previous works [7], [9], we combine a boundary-integral equation for electromagnetic scattering with a Creamer nonlinear surface. In order to reduce the numerical computation time and the required central memory to acceptable values, additional approximations will be done on both the electromagnetic and the hydrodynamic models. An approximate electromagnetic boundary-integral equation is considered, the small-slope integral equation (SSIE) recently developed by Saillard and 
Sentenac [13]. For the hydrodynamic model, we expand the Creamer formalism in a perturbative series up to the second order, restricting the model to light winds (typically up to $5 \mathrm{~m} / \mathrm{s}$ measured at $10-\mathrm{m}$ height). The first-order term corresponds to a linear surface, and is thus carried out using the spectral method on a semiempirical sea surface spectrum. However, the influence of the nonlinear hydrodynamic effects increase with radar frequency and led us to undress this spectrum [14] such that superimposing the second-order term makes it match the original one. Note that this was not necessary with HF and VHF radars, since the longer surface waves are hardly modified. We present simulations on a directional Pierson-Moskowitz (PM) spectrum for wind speeds ranging from 2 to $4 \mathrm{~m} / \mathrm{s}$ and for various values of the wind direction. At this stage, the influence of the shape of the coast or of superposition of swell is not considered. Effect on the nonlinearity of the surface on the Doppler spectrum is investigated, and the sensitivity of the model to wind speed and direction is studied.

Experimental data have shown that horizontal $(\mathrm{HH})$ and vertical (VV) Doppler spectra may exhibit strongly different shapes, in particular at grazing angles [15]-[21]. This has been interpreted as the manifestation of bound waves and/or nonBragg scattering effects due to, e.g., breaking waves [17]. In this paper, both vertical and horizontal polarizations are considered for the incident and scattered field, and differences between polarizations are outlined. Our motivation for computing the cross-polarized components comes from our wish of vanishing the single-scattering contribution, which strongly dominates the copolarized signature at low winds, to clearly exhibit higher order contributions.

This paper is organized as follows. In Section II, the outlines of the hydrodynamic model, starting from the general principle of the spectral method, are described. We focus first on the linear surface that is the comparative reference to our nonlinear model. Then, we present the nonlinear model based on the Creamer model and the technical aspects of the sea surface simulation with regards to the required computation cost and the physical processes. The section ends with the formulation of the undressed spectrum. Section III presents the electromagnetic model, while Section IV is devoted to results and comments. The paper ends with a section for concluding remarks and perspectives.

\section{Ocean Surface Model}

A modeling L-band radar Doppler spectra from the ocean requires an accurate description of the sea surface motion. One of the main difficulties lies in the fact that the geometry of the sea surface is of complex character, involving nonlinear wave interactions and random aspect.

The general solution of the sourceless linearized hydrodynamic equations is a linear sum of independent harmonic waves with undetermined amplitudes and propagating in accordance with the dispersion relation of free waves. A linear superposition of harmonic waves of which amplitude is equal to a Rayleigh-distributed random value times the square root of the sea surface spectrum with random uniformly distributed phase provides what is referred to as a linear sea surface. Motion of such a sample is easily derived from the dispersion relation. This method of surface sample generation is called the spectral method.

However, it is now well established that interactions between harmonic waves cannot be neglected if one aims at interpreting radar Doppler spectra. The usual way of describing the sea surface motion consists in combining a perturbative approach with the spectral method described above. A linear surface is first generated and higher order corrections are obtained from expansion of hydrodynamic formulas in terms of wave interactions. Such terms fill the lack of phase relationship between various waves, which is known to be the signature of the nonlinear character. However, keeping in mind that all sea surface spectra are of semiempirical nature, thus take all wave interactions into account, this approach implicitly assumes that higher order terms do not significantly modify the part of the surface spectrum that contributes to the radar echo. This is true up to the VHF radar frequency range for which the method has yielded satisfactory results, but the assumption is no longer valid at higher frequencies.

In L-band remote sensing, it appears that if the linear surface is generated from the complete sea surface spectrum, the second-order term would create a significant roughness bias in the submetric wavelength range. Therefore, the spectrum describing the linear part of the surface has to be built such that adding higher order terms leads to the chosen semiempirical surface spectrum. In the following, the spectrum of the linear surface will be called the undressed spectrum.

\section{A. Spectrum Function of Sea Surface}

We use a spectrum function

$$
P(\mathbf{k})=\psi_{\mathrm{PM}}(k) \phi(\theta)
$$

constituted by a PM omnidirectional spectrum

$$
\psi_{\mathrm{PM}}(k)=\frac{\alpha}{k^{4}} \exp \left(-\frac{5}{4}\left(\frac{k}{k_{\mathrm{p}}}\right)^{2}\right)
$$

and a spreading function

$$
\phi(\theta)=\mathcal{N}\left|\cos ^{5}\left(\frac{\theta-\theta_{\mathrm{v}}}{2}\right)\right|
$$

where $\mathbf{k}$ is the spatial wave vector of polar coordinates $(k, \theta)$. The PM spectrum depends on two parameters $\alpha=4.0510^{-3}$ and the spectrum peak wavenumber $k_{\mathrm{p}}$ which is function of the wind speed (see Table III for numerical values). In the spreading function expression, the wind orientation angle $\theta_{\mathrm{v}}$ denotes the direction from where the wind is blowing, following the standard definition used in meteorology. In all simulations, the horizontal projection of the incident electromagnetic wave vector has a null polar angle. Thus, $\theta_{\mathrm{v}}=0$ means that the radar is looking upwind. $\mathcal{N}=1 / \int_{-\pi}^{+\pi} \cos ^{5}(\theta / 2) d \theta=15 / 16$ is a normalization factor. 
TABLE I

Statistics of a Generated SuRfaCE OF AREA $100 \lambda \times 100 \lambda$ AND $N=512 \times 512$ SAMPLING POINTS FOR A 3-m/s WIND SPEED $\left(k_{\mathrm{p}}=0.73 \mathrm{rad} / \mathrm{s}\right)$ AND A WIND DIRECTION $\theta_{\mathrm{v}}=0^{\circ}$

\begin{tabular}{||c|c|c|c||}
\hline \multirow{2}{*}{ Surface } & \multicolumn{3}{|c||}{ Root mean square } \\
\cline { 2 - 4 } & Height & Slope $(x)$ & Slope $(y)$ \\
\hline Linear & $55.25 \mathrm{~mm}$ & 0.102 & 0.081 \\
Creamer(2) & $55.50 \mathrm{~mm}$ & 0.146 & 0.112 \\
\hline
\end{tabular}

\section{B. Linear Simulation}

As said above, we adopt in this paper the spectral method under the spatially homogeneous and time-stationary hypothesis. All the Fourier components of the linear surface are completely independent with random phases. This implies Gaussian statistics for the water height and all its derivatives. Formulation of such a surface is

$$
\eta(\mathbf{x}, t)=\int_{\mathbf{k}} \int_{\omega} A(\mathbf{k}, \omega) \exp i(\mathbf{k} \cdot \mathbf{x}-\omega t)
$$

where $\eta(\mathbf{x}, t)$ is the sea surface elevation at spatial location $\mathbf{x}$ for the time $t$. Also, the complex amplitude $A(\mathbf{k}, \omega)$ would be taken as function of the square root of the spectrum formula $P(\mathbf{k})$. For gravity waves, formula (4) is completed by a dispersion relation $\omega^{2}=g k$, and time dependence becomes trivial. We simulate a linear sea surface by discretizing the formula (4). The simulated sea surface elevation is taken as the real part of the Fourier transform in (4) according to the dispersion relation. Discretized forms of the simulation write as

$$
\eta_{t}(\mathbf{x})=\operatorname{Re} \sum_{\mathbf{k}} A_{t}(\mathbf{k}) e^{i \mathbf{k} \cdot \mathbf{x}}
$$

where the complex amplitude

$$
A_{t}(\mathbf{k})=\gamma(\mathbf{k}) \sqrt{2 P(\mathbf{k}) \delta k_{x} \delta k_{y}} e^{-i \omega t}
$$

and $\gamma$ is a complex Gaussian process with zero mean and unity standard deviation.

The sum (5) can be efficiently performed by inverse fast Fourier transform (FFT): $\eta_{t}=\operatorname{Re} \mathcal{F}^{I}\left[A_{t}\right]$. The discretization steps $\delta k_{x}=2 \pi / L_{x}$ and $\delta k_{y}=2 \pi / L_{y}$ are thus related to the dimensions or periods $L_{x}$ and $L_{y}$ of the surface. The generation of one linear surface with $N$ sampling points involves a number of floating-point operations of order $N \log N$.

In order to check the simulation, we compute some statistical characteristics of the generated surface. The root mean square (rms) values of height and slopes in both directions $x$ and $y$ are written in Table I. Length and area in the simulation are expressed in electromagnetic wavelength units (here $\lambda=$ $25 \mathrm{~cm}$ ). Of interest is that the rms height value computed directly from the PM formula gives $0.221 \lambda$ and then appears to compare well with values in Table I.

\section{Nonlinear Simulation}

An approach to carry out nonlinear models involves perturbation techniques around the water surface level at rest
TABLE II

Characteristics of Nonlinear SuRface Generation Methods

\begin{tabular}{||c|c|c|c||}
\hline Method & Hamiltonian & order & numerical cost \\
\hline Barrick-Weber & complete & 2 & $N^{2}$ \\
Creamer & truncated & $\infty$ & $N^{2}$ \\
Creamer (2) & truncated & 2 & $N \log N$ \\
\hline
\end{tabular}

to determine the higher order corrections to the linearized solution. This was used successfully in remote sensing in the past by different authors, in particular Valenzuela [22]. The general principle of the perturbation technique is that higher order terms are nonlinear functions of the linearized solution. However, implementation of these models is of high numerical cost $\left(N^{2}\right)$, preventing their use for a Monte Carlo simulation on 2-D surfaces.

Another way to simulate nonlinear effect is the Hamiltonian formalism under the weak wave-turbulence theory, of which extensive applications have been made in the fields of water surface waves since the fundamental work of Zakharov [23]. In this study, we will make use of a recent formulation of the Hamiltonian formalism as given by [8], also considered by Toporkov and Brown for 1-D sea surfaces [9].

The Creamer formulation writes as a nonlinear transformation of the Hilbert transform of the linear surface. In 2-D, this Hilbert transform is defined as a vector. At a given time $t$, its expression, derived from (4), is

$$
\mathbf{h}_{t}(\mathbf{x})=\operatorname{Re} \sum_{\mathbf{k}}\left(-i \frac{\mathbf{k}}{k}\right) A_{t}(\mathbf{k}) e^{i \mathbf{k} \cdot \mathbf{x}} .
$$

The Hilbert transform can be computed by FFT, at a $N \log N$ cost.

The Creamer nonlinear transform writes

$$
C_{t}(\mathbf{k})=\frac{1}{N} \sum_{\mathbf{x}} \frac{\exp \left(i \mathbf{k} \cdot \mathbf{h}_{t}(\mathbf{x})\right)-1}{k} e^{-i \mathbf{k} \cdot \mathbf{x}} .
$$

However, this transform cannot be computed by FFT, since the term $\exp \left(i \mathbf{k} \cdot \mathbf{h}_{t}(\mathbf{x})\right)$ depends on both $\mathbf{k}$ and $\mathbf{x}$. At last, the Creamer method reveals to have also a $N^{2}$ numerical cost. To circumvent these difficulties, we expand the exponential operator as a series. Then, the nonlinear transform writes $C_{t}=$ $\sum_{n \geq 1} C_{t}^{n}$, with

$$
C_{t}^{n}(\mathbf{k})=\frac{1}{N} \sum_{\mathbf{x}} \frac{\left(i \mathbf{k} \cdot \mathbf{h}_{t}(\mathbf{x})\right)^{n}}{n ! k} e^{-i \mathbf{k} \cdot \mathbf{x}} .
$$

One can verify that the first order of this series $C_{t}^{1}$ identifies with $A_{t}$, that is, with the linear surface, when the second order is given by

$$
C_{t}^{2}=-\frac{k_{x}^{2}}{2 k} \mathcal{F}^{D}\left[h_{t_{x}}^{2}\right]-\frac{k_{x} k_{y}}{k} \mathcal{F}^{D}\left[h_{t_{x}} h_{t_{y}}\right]-\frac{k_{y}^{2}}{2 k} \mathcal{F}^{D}\left[h_{t_{y}}^{2}\right] .
$$

Therefore, a second-order Creamer surface can be obtained by $\eta_{t}=\operatorname{Re} \mathcal{F}^{I}\left[A_{t}+C_{t}^{2}\right]$. Table II summarizes the characteristics of the Creamer method. 
TABLE III

PARAMETERs OF THE Undressed SPECTRUM Against THE Wind SpeED

\begin{tabular}{||c|c|c|c||}
\hline Wind speed $(\mathrm{m} / \mathrm{s})$ & $k_{p}(\mathrm{rad} / \mathrm{m})$ & $k_{c}(\mathrm{rad} / \mathrm{m})$ & $p$ \\
\hline 2 & 1.64 & 8.0 & 4.30 \\
3 & 0.73 & 8.0 & 4.85 \\
4 & 0.41 & 4.0 & 4.85 \\
\hline
\end{tabular}

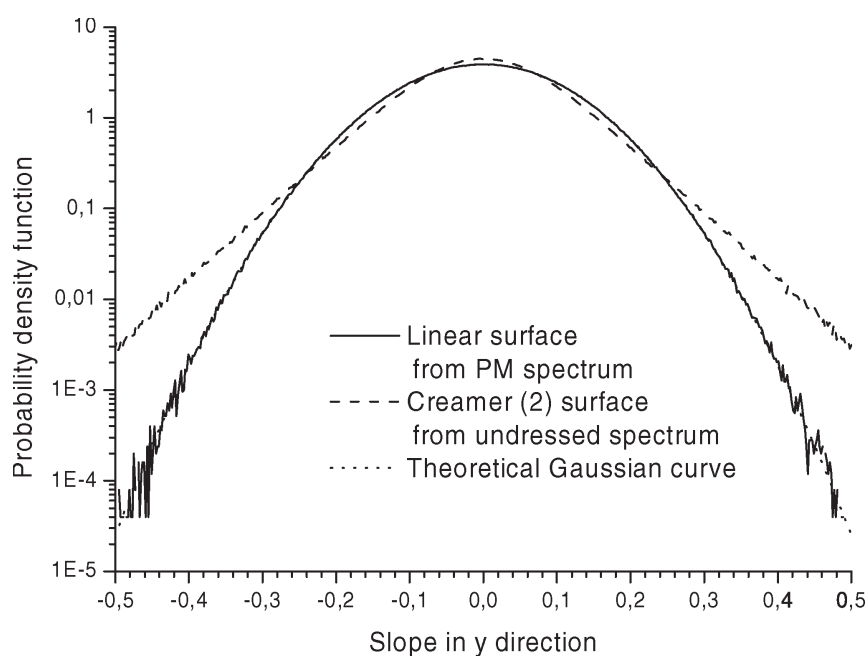

Fig. 1. Slopes probability distribution functions in the $y$ direction computed over 48 surface samples.

Statistics of the linear and Creamer (2) simulations can be compared in Table I. The rms height remains unchanged, but the slopes are strongly increased, about one half. This indicates an unrealistic magnification of the small-scale roughness, which leads in Section IV-A to an overestimation of the cross section (Fig. 3).

\section{Undressed Spectrum}

In order to correct this artifact about small-scale behavior, one must use the undressed spectrum as an input of the model instead of the semiempirical sea surface spectrum. In our opinion, there is no clear method in the hydrodynamic theory to undress one of the semiempirical sea spectra available in the literature. The present procedure is thus empirical. To prevent the unrealistic magnification of small-scale roughness in the Creamer (2) surface, we propose an undressed spectrum $\psi_{u}$ with reduced small-scale roughness. The Creamer (2) transform of a surface generated by the spectral method applied to the undressed spectrum is called a Creamer (2) undressed surface

$$
\psi_{u}(k)= \begin{cases}\psi_{\mathrm{PM}}(k), & k<k_{\mathrm{c}} \\ \beta k^{-p}, & k>k_{\mathrm{c}}\end{cases}
$$

Coefficient $\beta=k_{\mathrm{c}}^{p} \psi_{\mathrm{PM}}\left(k_{\mathrm{c}}\right)$ ensures the continuity of the undressed spectrum at wavenumber $k=k_{\mathrm{c}}$. The two parameters $k_{\mathrm{c}}$ and $p$ have been determined numerically for different values of the wind speed (Table III) so that the Creamer (2) undressed surfaces possess the same height and slopes root mean squares as the linear PM surface. As one can see, the undressed spectrum decreases faster than the PM spectrum for wavenumbers higher than $k_{\mathrm{c}}$.
The linear PM surfaces and the Creamer (2) undressed surfaces have different probability distribution functions. The slope distributions in the $y$ direction for the two surfaces is represented in Fig. 1. By definition, the linear PM surface slopes are Gaussian. Being nonlinear, the Creamer (2) surface is also non-Gaussian.

Note that this undressed spectrum, rather than the complete PM spectrum, should be used in perturbative models like SPM2 at L.

\section{Electromagnetic Scattering Model}

Rino et al. [7] (see also Toporkov and Brown [9]) have shown how a frequency-domain integral-equation-based numerical method can be applied to time-varying surfaces. However, a deterministic ocean Doppler spectrum requires the computation of some hundreds of time-harmonic scattered fields, one for each time step, and statistical results are typically obtained by averaging over 100 Doppler spectra. Therefore, one understands that such a simulation is numerically intensive, and is made possible for 2-D surfaces only with a fast harmonic integral equation method.

Following the boundary-integral formalism, the tangential components of the fields on the surface are the two unknowns of the scattering problem. The Stratton-Chu equations for the lower medium state that the relationship between the tangential components of the electric and magnetic fields, the surface impedance, is an integral relationship. For highly reflecting materials (HRIEs) like the ocean surface at microwave frequency, the lower medium Green's function shows a fast, exponential decreasing behavior. The surface impedance is thus a very short-range integral relationship, and can be assumed to be local. This local impedance has been derived by Marvin and Celli [24] and depends on the local curvatures of the surface.

The four Stratton-Chu equations include integro-differential operators with hypersingular kernels [25]. However, these equations can be linearly combined in order to produce two nonhypersingular equations. The surface impedance can be inserted into one of these equations to produce a single nonhypersingular integral equation for HRIEs [13], [26]. This equation is an extension of the magnetic field integral equation for nonperfectly conducting surfaces. It has similar singularity, and can be numerically solved through the same techniques.

The HRIE can be solved without supplementary approximation by use of the sparse matrix flat surface iterative approach [27]. However, the computing time scales as $N^{2}$, where $N$ is the number of surface unknowns. And the method is very random access memory (RAM) demanding for surfaces with a large correlation length such as the ocean surface.

We take a further step toward the ocean surface in [28]. In this paper, the sparse matrix flat surface iterative approach is simplified by considering the Meecham-Lysanov approximation [29]. In the boundary-integral formalism, the interaction between two points of the surface is modelized by the Green's function and its derivatives. The Green's function itself is a function of the distance between the interacting points. The Meecham-Lysanov approximation consists in neglecting the height difference $z$ in the distance $r=\sqrt{d^{2}+z^{2}}$. When the equation is cast into a matrix-vector form, the interaction 


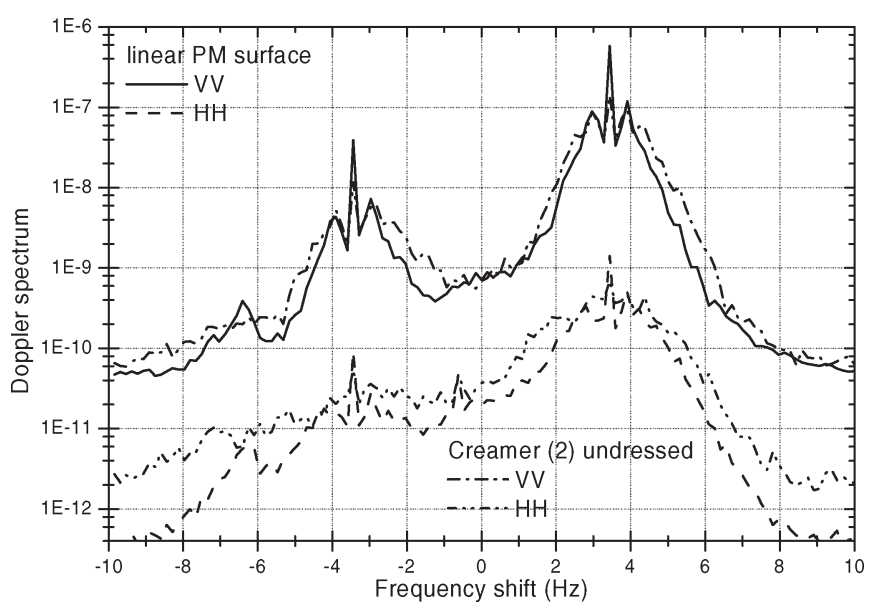

Fig. 2. Comparison between the linear PM and the Creamer (2) undressed surface copolarized Doppler spectrum. Electromagnetic frequency is $1.2 \mathrm{GHz}$, incidence is $70^{\circ}$, wind speed is $3 \mathrm{~m} / \mathrm{s}$, and wind direction is $60^{\circ}$.

matrix reveals a 2-D Toeplitz structure. The linear system is solved iteratively, and the matrix-vector product is performed by 2-D FFTs. The computing time and RAM required by the method scale, respectively, as $N \log N$ and $N$.

This approximation is a small-slope approximation since a small height difference $z$ compared to the horizontal distance $d$ corresponds to a small slope: $z / d=p \ll 1$. Our method is called the SSIE. Its theoretical validity domain is $K \sigma s \ll 1$, where $K$ denotes the electromagnetic wavenumber in vacuum, $\sigma$ and $s$ are, respectively, the height and slope root mean squares of the surface. Numerical experiments have shown in [28] that this domain can be extended for copolarized scattering to values of the product $K \sigma s$ equal and even slightly superior to one.

The incident field is a Gaussian beam. For grazing angles of incidence, the minimum enlightened 2-D surface scales as $\theta_{g}^{-3}$, with $\theta_{g}=\pi / 2-\theta_{i}$ being the grazing angle (see the Appendix). The number of surface unknowns $N$ evolves in the same way.

\section{NumericAl Results}

At the working electromagnetic frequency of $1.2 \mathrm{GHz}(\lambda=$ $0.25 \mathrm{~m})$, the ocean complex relative permittivity is $\varepsilon=73.5+$ $i 61.0$ for average values of the sea surface temperature and salinity [30]. For a $4-\mathrm{m} / \mathrm{s}$ wind speed, the peak wavelength $\lambda_{\mathrm{p}}=2 \pi / k_{\mathrm{p}}$ is around $15 \mathrm{~m}$. Samples are square surfaces of $100 \lambda=25 \mathrm{~m}$ sides, sampled with 512 points in each direction, and incidence is set to the maximum value for such a surface length, that is $70^{\circ}$. The Doppler computation is realized with $N_{t}=128$ time steps of $\delta t=40 \mathrm{~ms}$. The Monte Carlo average is performed over 48 Doppler spectrum samples. Note that no additional Doppler shift due to the wind drift current of the water surface is taken into account in the presented simulations.

\section{A. Effects of the Surface Nonlinearities}

For this study of the effects of the surface nonlinearities on the Doppler spectrum, the wind speed is set to $3 \mathrm{~m} / \mathrm{s}$, and the wind direction is $60^{\circ}$ from the plane of incidence.

Fig. 2 compares the Doppler spectrum for a nonlinear surface, derived by applying the Creamer (2) transform to surfaces generated from the undressed PM spectrum (10), with that from

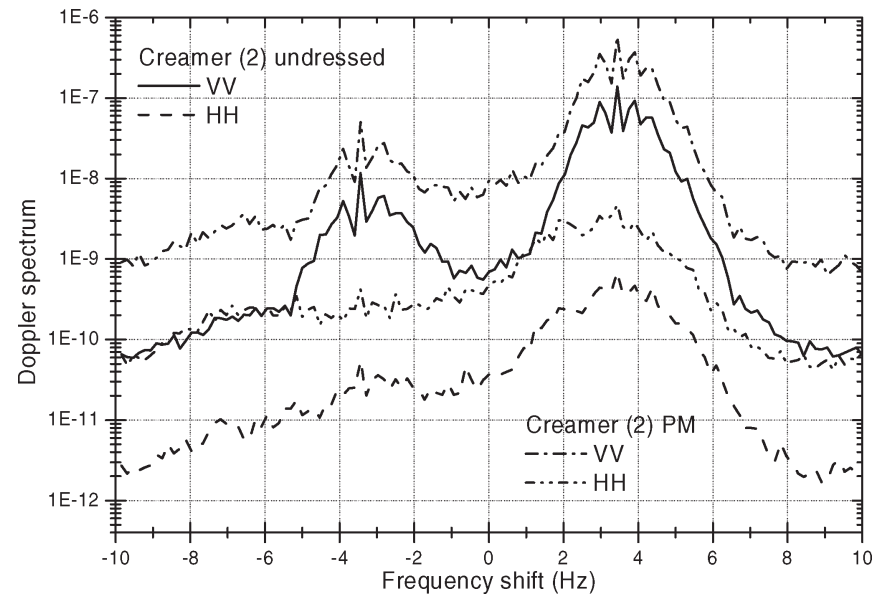

Fig. 3. Comparison between copolarized Doppler spectra derived from PM surface spectrum and the undressed surface spectrum. Electromagnetic frequency is $1.2 \mathrm{GHz}$, incidence is $70^{\circ}$, wind speed is $3 \mathrm{~m} / \mathrm{s}$, and wind direction is $60^{\circ}$

the linear PM surface. For the sake of clarity, only copolarized components are shown. The two Doppler spectra are close to each other, but significant differences have to be noticed. The most obvious effect of nonlinearities is the increase of the level of Doppler spectra for all frequency shifts except at Bragg frequency (with one exception for vertical polarization in the vicinity of $-6 \mathrm{~Hz}$ ). It is interesting to notice that for a linear surface the Bragg lines predicted by SPM at first order clearly appear at $\pm f_{\mathrm{B}}$ with $f_{\mathrm{B}}=\sqrt{g \sin \theta^{i} / \pi \lambda} \simeq 3.4 \mathrm{~Hz}$. The decrease of their amplitude is directly linked to the decrease of the amplitude of Bragg wave when undressing the linear wave spectrum $\psi_{\mathrm{PM}}$. This is obvious for VV polarization where the maxima are $6 \mathrm{~dB}$ below, which is exactly $10 \log \left(\psi_{\mathrm{PM}}\left(k_{\mathrm{B}}\right) / \psi_{u}\left(k_{\mathrm{B}}\right)\right)$. For $\mathrm{HH}$ polarization, the maxima of Bragg lines are lowered by $3 \mathrm{~dB}$ only because it comes with a $3-\mathrm{dB}$ overall increase of the Doppler spectrum. Let us recall that for HH spectra, the behavior strongly depends on incidence angle, especially at grazing, when fast scatterers may become the main contributors [12].

These results are also consistent with remote-sensing experiments performed either in the microwave range [15] or in VHF range with big waves [31], which have shown that for such a rms-height-to-electromagnetic-wavelength ratio, Bragg lines are of the same order of magnitude as the secondary peaks which appear, especially in VV polarization, at $f= \pm f_{\mathrm{B}} \pm f_{\mathrm{p}}$, with $f_{\mathrm{p}}=\sqrt{g k_{\mathrm{p}}} / 2 \pi \simeq 0.4 \mathrm{~Hz}$. These secondary peaks are also well known in ocean remote sensing, since they are predicted by the second-order small perturbation theory and observed with lower radar frequencies. Within the frame of this perturbative approach, the decreasing behavior of the side lobes away from Bragg lines is closely related to the decrease of the surface spectrum $\left(f^{-5}\right.$ here). Here, significant discrepancies from such predictions are observed, due to the contribution of higher order interactions on one hand, to the size of the incident beam [31] on the other hand.

It is interesting to note that undressing the spectrum mainly results in lowering the cross section, with minor changes of the shape of Doppler spectra. As shown in Fig. 3, the shift in VV varies from $6 \mathrm{~dB}$ around Bragg frequency up to $10 \mathrm{~dB}$ away from it, while the difference is even stronger in $\mathrm{HH}$, from 8 


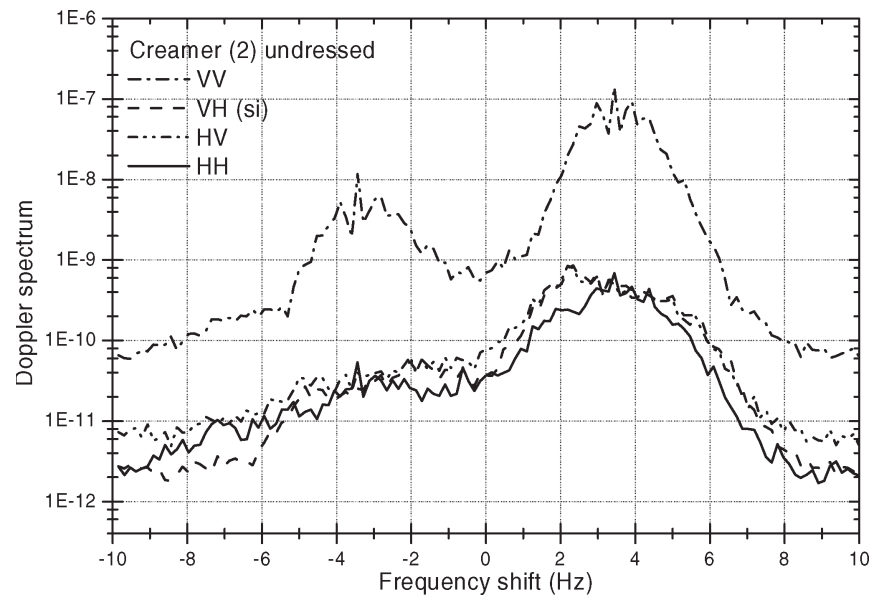

Fig. 4. Creamer (2) undressed surface co- and cross-polarized Doppler spectrum. Electromagnetic frequency is $1.2 \mathrm{GHz}$, incidence is $70^{\circ}$, wind speed is $3 \mathrm{~m} / \mathrm{s}$, and wind direction is $60^{\circ}$.

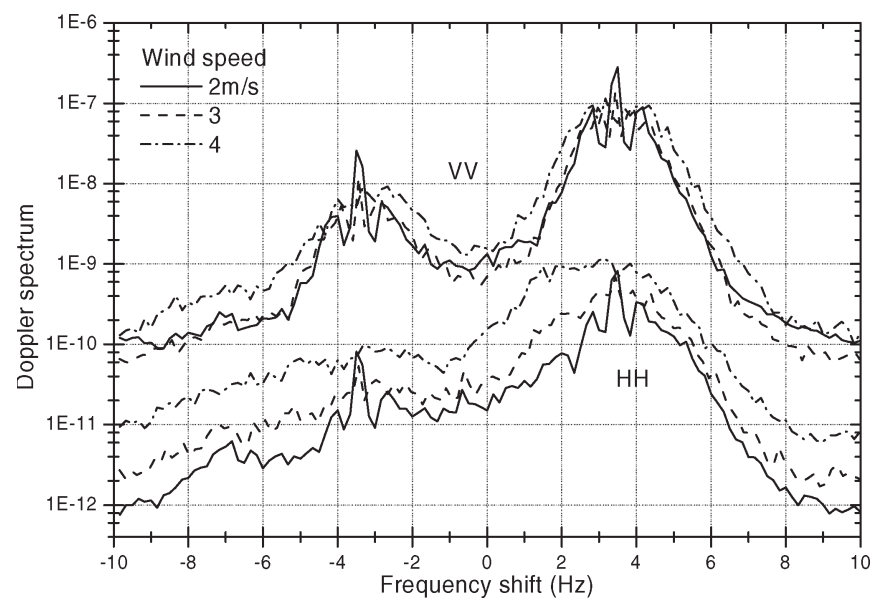

Fig. 5. Sensitivity of the ocean copolarized Doppler spectrum to the wind speed. Electromagnetic frequency is $1.2 \mathrm{GHz}$, incidence is $70^{\circ}$, and wind direction is $60^{\circ}$.

to $15 \mathrm{~dB}$, respectively. Fig. 4 shows all four components of the Creamer (2) undressed surface Doppler spectrum. Since only multiple scattering contributes to cross polarization, no Bragg line occurs in HV and VH. It also appears that the cross-polarized and horizontal-copolarized components are of comparable level. In [32], this was predicted to occur at L-band above $1-\mathrm{m} / \mathrm{s}$ wind speed and for "large" incidence angles.

\section{B. Sensitivity to Wind Speed and Direction}

To be used in an inversion process, a forward model has to translate accurately the influence of the geophysical parameters of interest on the data. This section does not pretend to constitute a quantitative parametrical study, but aims at showing that the present model behaves as expected with wind characteristics, its speed and its direction.

Fig. 5 shows the copolarized Doppler spectrum for both V and $\mathrm{H}$ incident fields and for three values of the wind speed: 2 , 3 , and $4 \mathrm{~m} / \mathrm{s}$ at a $10-\mathrm{m}$ height. Wind direction is set to $60^{\circ}$ from the plane of incidence.

Fig. 5 indicates that between 2 and $3 \mathrm{~m} / \mathrm{s}$, wind speed has noticeable impact on the VV Doppler spectrum mainly at Bragg

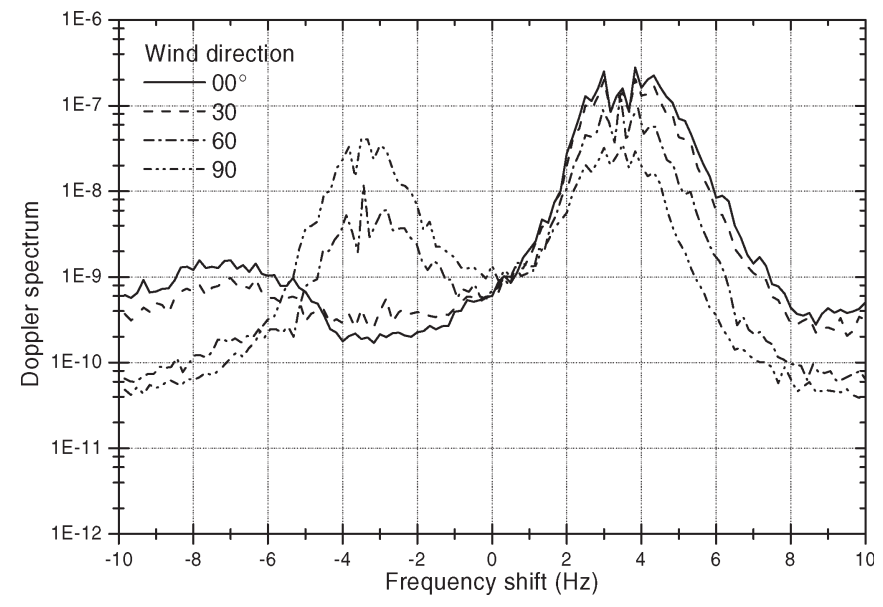

Fig. 6. Sensitivity of the ocean VV Doppler spectrum to the wind direction. Electromagnetic frequency is $1.2 \mathrm{GHz}$, incidence is $70^{\circ}$, and wind speed is $3 \mathrm{~m} / \mathrm{s}$.

frequency, while in $\mathrm{HH}$ Bragg line remains almost constant and the remaining is increased by 2 to $3 \mathrm{~dB}$. As expected, Bragg lines progressively disappear when wind speed increases. Up to $3-\mathrm{m} / \mathrm{s}$ wind speed, the secondary peaks at $\pm f_{\mathrm{B}} \pm f_{\mathrm{p}}$ can be detected and translate accurately the increase of the wavelength of the dominant wave. Between 3 and $4 \mathrm{~m} / \mathrm{s}$, wind speed increase induces an additional broadening of the lobes, both in $\mathrm{VV}$ and HH. This is the signature of a strong increase of higher order contributions, probably linked to the increase of the rms height of the surface profile from $\lambda / 4$ up to $\lambda / 2$.

Fig. 6 shows the copolarized Doppler spectrum for a vertically polarized incident field for four representative values of the wind direction: $0^{\circ}$ (upwind), $30^{\circ}, 60^{\circ}$, and $90^{\circ}$ (crosswind). Wind speed is set to $3 \mathrm{~m} / \mathrm{s}$ at a $10-\mathrm{m}$ height.

Note that the spreading function (3) vanishes in the downwind direction and that first-order perturbation theory predicts a difference between Bragg lines of $28 \mathrm{~dB}$ at $30^{\circ}$ and $12 \mathrm{~dB}$ at $60^{\circ}$. This is very close to what is computed in VV. Whether such a spreading function is realistic or not can be discussed, but the point here is that the present model permits to derive some properties of the spreading function, especially in the decimetric range. The influence of the spreading function on the shape of Doppler spectra is left for future work.

\section{CONClusion}

It has been shown that the combination of the SSIE method for the electromagnetic scattering with a Creamer (2) model for describing a time-evolving wind-driven sea surface permits us to compute, at reasonable numerical cost, realistic full-polarized ocean Doppler spectra in L-band. The use of Creamer's perturbative approach restricts this 3-D model to light winds. In addition, the Creamer model cannot be directly applied to a semiempirical ocean spectrum at microwave frequencies, since it would induce unrealistic magnification of small-scale roughness that mainly contributes to the radar cross section. Therefore, we have proposed an empirically undressed PM spectrum for wind speed values of 2, 3, and $4 \mathrm{~m} / \mathrm{s}$, which corrects this artifact.

Simulations of Doppler spectra at L-band $(\lambda=0.25 \mathrm{~m})$ and $70^{\circ}$ incidence, for 2-, 3-, and 4- $\mathrm{m} / \mathrm{s}$ wind speeds and different 
wind directions have been presented. The model has been successfully used to investigate the effect of the hydrodynamic nonlinearities, and the sensitivity of the Doppler spectrum to wind speed and direction. Copolarized components, especially $\mathrm{VV}$, share some characteristics with small perturbation theory: the presence of two Bragg lines, each one shouldered by two secondary peaks. It can be concluded that a global shift of a Doppler spectrum would be detected through the shift of Bragg lines and that such an L-band Doppler radar may be useful to map surface currents. Compared to HF radars, which take benefit from surface waves, the range at L-band will be much lower but the experimental setup is much more flexible and the resolution can be improved. Therefore, such an L-band Doppler radar could be helpful for coastal current studies. To this end, it would also be important to take into account superposition of long swell to short wind waves. This is possible at moderate cost by modeling nonlinear hydrodynamic interactions as those of short waves with a surface current. In its present form, the model cannot deal with low grazing incidence angles, for numerical reasons related to memory requirements and computation accuracy, since the radar cross section decreases as the fourth power of the grazing angle.

\section{APPENDIX \\ Gaussian InCIDENT BeAm at GRAZING ANGLE}

A Gaussian beam is spectrally characterized by a mean direction $\left(k_{i}, 0\right)$, where $k_{i}=K \sin \theta_{i}$ and $\theta_{i}$ is the incidence angle, and dimensions $\sigma_{k_{x}}, \sigma_{k_{y}}$. The Gaussian function is not compact, but one can set a parameter $n_{k}$ and limit the spectral domain of a Gaussian beam in the plane $\left(k_{x} O k_{y}\right)$ to an ellipse of center the mean direction and half axis $a_{x}=$ $(1 / 2) n_{k} \sigma_{k_{x}}$ and $a_{y}=(1 / 2) n_{k} \sigma_{k_{y}}$. A similar reasoning can be done in the spatial domain. The footprint of a Gaussian beam is elliptical, and can be inserted in a rectangle of dimensions $L_{x}=n_{r} \sigma_{x}$ and $L_{y}=n_{r} \sigma_{y}$. Spatial and spectral dimensions are linked by $\sigma_{x} \sigma_{k_{x}}=\sigma_{y} \sigma_{k_{y}}=1$. The Gaussian beam is only constituted of propagative plane waves. The ellipse $\left(\left(k_{x}-\right.\right.$ $\left.\left.k_{i}\right) / a_{x}\right)^{2}+\left(k_{y} / a_{y}\right)^{2}=1$ should thus be included into the circular propagative plane wave boundary $k_{x}^{2}+k_{y}^{2}=K^{2}$. One easily finds $a_{x} \leq K-k_{i}$, and for $a_{x}=K-k_{i}, a_{y}$ must verify $a_{y} \leq \sqrt{K\left(K-k_{i}\right)}$. The minimum surface dimensions are thus

$$
\left\{\begin{array}{l}
L_{x}=\frac{n_{r} n_{k}}{2 K} \frac{1}{1-\sin \theta_{i}} \\
L_{y}=\frac{n_{r} n_{k}}{2 K} \frac{1}{\sqrt{1-\sin \theta_{i}}} .
\end{array}\right.
$$

Parameters $n_{r}$ and $n_{k}$ are commonly set to 8 . At the limit of grazing angles, this gives a sampled area that scales as

$$
L_{x} L_{y} \rightarrow \frac{1}{\sqrt{2}}\left(\frac{n_{r} n_{k}}{2 K}\right)^{2} \theta_{r}^{-3} .
$$

Note that $n_{r}$ and $n_{k}$ may depend on $\theta_{r}$ at grazing.

\section{REFERENCES}

[1] D. D. Crombie, "Doppler spectrum of sea echo at $13.56 \mathrm{Mc} / \mathrm{s}$," Nature, vol. 175, no. 4459, pp. 681-682, Apr. 1955.

[2] J. W. Wright, "A new model for sea clutter," IEEE Trans. Antennas Propag., vol. AP-16, no. 2, pp. 217-223, Mar. 1968.
[3] F. G. Bass, I. M. Fuks, A. I. Kalmykov, I. E. Ostrovsky, and A. D. Rosenberg, "Very high frequency microwave scattering by a disturbed sea surface," IEEE Trans. Antennas Propag., vol. AP-16, no. 5, pp. 554-568, Sep. 1968.

[4] D. E. Barrick and B. L. Weber, "On the nonlinear theory for gravity waves on the ocean's surface. Part II: Interpretation and applications," J. Phys. Oceanogr., vol. 7, no. 1, pp. 11-21, 1977.

[5] B. L. Weber and D. E. Barrick, "On the nonlinear theory for gravity waves on the ocean's surface. Part I: Derivations," J. Phys. Oceanogr., vol. 7, no. 1, pp. 3-10, 1977.

[6] R. R. Lentz, "A numerical study of electromagnetic scattering from oceanlike surface," Radio Sci., vol. 9, no. 12, pp. 1139-1146, Dec. 1974.

[7] C. L. Rino, T. L. Crystal, A. K. Koide, H. D. Ngo, and H. Guthard, "Numerical simulation of backscatter from linear and nonlinear ocean surface realizations," Radio Sci., vol. 26, no. 1, pp. 51-71, 1991.

[8] D. B. Creamer, F. Henyey, R. Schult, and J. Wright, "Improved linear representation of ocean surface waves," J. Fluid Mech., vol. 205, pp. 135-161, 1989.

[9] J. V. Toporkov and G. S. Brown, "Numerical simulations of scattering from time-varying, randomly rough surfaces," IEEE Trans. Geosci. Remote Sens., vol. 38, no. 4, pp. 1616-1624, Jul. 2000.

[10] —, "Numerical study of the extended Kirchhoff approach and the lower order small slope approximation for scattering from ocean-like surfaces: Doppler analysis," IEEE Trans. Antennas Propag., vol. 50, no. 4, pp. 417-425, Apr. 2002.

[11] J. T. Johnson, J. V. Toporkov, and G. S. Brown, "A numerical study of backscattering from time-evolving sea surfaces: Comparison of hydrodynamic models," IEEE Trans Geosci. Remote Sens., vol. 39, no. 11, pp. 2411-2420, Nov. 2001.

[12] A. R. Hayslip, J. T. Johnson, and G. R. Baker, "Further numerical studies of backscattering from time evolving nonlinear sea surfaces," IEEE Trans. Geosci. Remote Sens., vol. 41, no. 10, pp. 2287-2293, Oct. 2003.

[13] M. Saillard and A. Sentenac, "Rigorous solutions for electromagnetic scattering from rough surfaces," Waves Random Media, vol. 11, no. 3, pp. R103-R137, Jul. 2001.

[14] T. Elfouhaily, M. Joelson, S. Guignard, D. Thompson, B. Chapron, and D. Vandemark, "Analysis of random nonlinear water waves: The Stokes-Woodward technique," C. R. Mécanique, vol. 331, no. 3, pp. 189-196, Mar. 2003.

[15] P. H. Y. Lee, J. D. Barter, K. L. Beach, C. L. Hindman, B. M. Lake, H. R. J. C. Shelton, A. B. Williams, R. Yee, and H. C. Yuen, "X band microwave backscattering from ocean waves," J. Geophys. Res., vol. 100, no. C2, pp. 2591-2611, Feb. 1995.

[16] P. H. Y. Lee, J. D. Barter, E. Caponi, M. Caponi, C. L. Hindman, B. M. Lake, and H. Rungaldier, "Wind speed dependence of smallgrazing-angle microwave backscatter from sea surfaces," IEEE Trans. Antennas Propag., vol. 44, no. 3, pp. 333-340, Mar. 1996.

[17] P. H. Y. Lee, L. Wang, and R. Yee, "What are the mechanisms for nonBragg scattering from water wave surfaces?," Radio Sci., vol. 34, no. 1, pp. $123-138,1999$.

[18] W. J. Plant, "A model for microwave Doppler sea return at high incidence angles: Bragg scattering from bound, tilted waves," J. Geophys. Res., vol. 102, no. C9, pp. 21131-21146, Sep. 1997.

[19] W. J. Plant, W. C. Keller, V. Hesany, T. Hara, E. Bocka, and M. A. Donelan, "Bound waves and Bragg scattering in a wind-wave tank," J. Geophys. Res., vol. 104, no. C2, pp. 3243-3264, Feb. 1999.

[20] A. D. Rozenberg, M. J. Ritter, W. K. Melville, C. C. Gottschall, and A. V. Smirnov, "Free and bound capillary waves as microwave scatterers: Laboratory studies," IEEE Trans. Geosci. Remote Sens., vol. 37, no. 2, pp. 1052-1065, Mar. 1999.

[21] M. A. Sletten, J. C. West, X. Liu, and J. H. Duncan, "Radar investigation of breaking waves at low grazing angles with simultaneous high speed optical imagery," Radio Sci., vol. 38, no. 6, p. 1110, 2003.

[22] G. R. Valenzuela, "The effect of capillarity and resonant interactions on the second-order Doppler spectrum of radar sea echo," J. Geophys. Res., vol. 79, no. 33, pp. 5031-5037, 1974.

[23] V. E. Zakharov, "Stability of periodic waves of finite amplitude on the surface of a deep fluid," J. Appl. Mech. Tech. Phys., vol. 9, no. 2, pp. 190-194, 1968.

[24] A. M. Marvin and V. Celli, "Relation between the surface impedance and the extinction theorem on a rough surface," Phys. Rev. B, vol. 50, no. 19, pp. 14 546-14 553, 1994.

[25] P. A. Martin and P. Ola, "Boundary integral equations for the scattering of electromagnetic waves by a homogeneous dielectric obstacle," Proc. $R$. Soc. Edinburgh, vol. 123A, pp. 185-208, 1993.

[26] G. Soriano and M. Saillard, "Scattering of electromagnetic waves from 
two-dimensional rough surfaces with impedance approximation," J. Opt. Soc. Amer. A, vol. 18, no. 1, pp. 124-133, Jan. 2001.

[27] K. Pak, L. Tsang, C. H. Chan, and J. T. Johnson, "Backscattering enhancement of electromagnetic waves from two-dimensional perfectly conducting random rough surfaces based on Monte Carlo simulations," J. Opt. Soc. Amer. A, vol. 12, no. 11, pp. 1491-1499, Nov. 1995.

[28] M. Saillard and G. Soriano, "Fast numerical solution for scattering from rough surfaces with small slopes," IEEE Trans. Antennas Propag., vol. 52, no. 10, pp. 2799-2802, Oct. 2004.

[29] A. G. Voronovich, Wave Scattering From Rough Surfaces. Berlin, Germany: Springer-Verlag, 1994.

[30] L. A. Klein and C. T. Swift, "An improved model for the dielectric constant of sea water at microwave frequencies," IEEE Trans. Antennas Propag., vol. AP-25, no. 1, pp. 104-111, Jan. 1977.

[31] Y. Hisaki and M. Tokuda, "VHF and HF sea echo Doppler spectrum for a finite illuminated area," Radio Sci., vol. 36, no. 3, pp. 425-440, 2001.

[32] G. R. Valenzuela, "Depolarization of EM waves by slightly rough surfaces," IEEE Trans. Antennas Propag., vol. AP-15, no. 4, pp. 552-557, Jul. 1967.

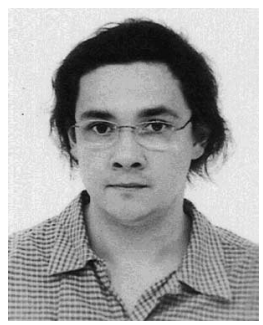

Gabriel Soriano was born in Paris, France, in 1972. He received the M.S. degree in physics and the Ph.D. degree in physics from the Paul Cezanne University, Marseille, France, in 1996 and 2000, respectively.

$\mathrm{He}$ is currently an Associate Professor in the Paul Cezanne University. He is a member of the electromagnetical and optical remote sensing (SEMO) team of the Fresnel Institute, Marseille, France. He works on surface scattering and associated numerical methods.

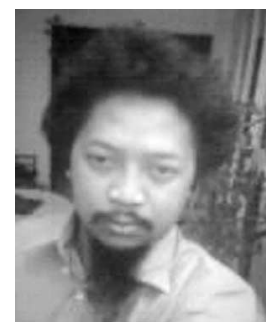

Maminirina Joelson received the M.S. degree in applied mathematics from the University of AixMarseille II, Marseille, in 1992, the postgraduate diploma in energetic mechanics from the University of Provence in 1993, and the Ph.D. degree in fluid mechanics from the Institut de Recherche sur les Phénomènes Hor Equilibre (IRPHE) Research Institute, Marseille, in 1997.

From 1998 to 2000, he was in a Postdoctoral position at the Interactions Océan-Atmosphère (IOA) team of IRPHE. From 2000 to 2003, he taught mathematics and thermodynamics at the French National Merchant Shipping School (Ecole Nationale de Marine Marchande, Marseille). In 2004, he worked with the SEMO team of the Fresnel Institute, Marseille, France. Since September 2004, he has been with the Climate Soil Environment Laboratory [Unité Mixte de Recherche (UMR)-Institut National de la Recherché Agronomique (INRA)] of the University of Avignon, where he is currently an Associate Professor in the Department of Physics. His current research interests include nonlinear dynamic stochastic and fluid diffusion in random porous media.

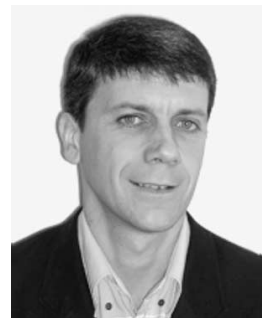

Marc Saillard (M'00-A'01-M'03) was born in Marseille, France, in 1961. He received the "Agrégation" degree in physics and the Ph.D. degree in physics from the University of Marseille, Marseille, France, in 1985 and 1990, respectively.

From 1990 to 1997, he was Assistant Researcher at the National Center for Scientific Research (CNRS) at the Electromagnetic Optics Laboratory. In 1997, he got a Professor position at the University of Marseille where he runs the remote-sensing group of the Institut Fresnel. In 2003, he moved to the University of Toulon, as a Professor, where he joined the remote-sensing laborarory [Laboratoire de sondages Electromagnetiques de l'environnement Terrestre (LSEET)]. His professional interests lie in surface and volume scattering, inverse scattering, and remote sensing. 